\title{
The Clinical Efficacy of Xiaoqinglong Decoction with Gypsum Decoction in Treating Pediatric Pneumonia
}

\author{
Dongdong Shen ${ }^{1, a}$, Jianghong Hou ${ }^{{ }^{1,}, b}$, Zhiqiao Dong ${ }^{1, c}$, Haijun Shan ${ }^{1, d}$, \\ Fei Yuan ${ }^{1, \mathrm{e}}$ \\ ${ }^{1}$ Henan Province Hospital of Traditional Chinese Medicine, Zhengzhou, Henan, 450002 \\ a email, ${ }^{\mathrm{b}}$ email, ${ }^{\mathrm{c}}$ email, ${ }^{\mathrm{d}}$ email, ${ }^{\mathrm{e}}$ email
}

\begin{abstract}
Keywords: Xiaoqinglong Decoction, Gypsum Decoction; Pneumonia in Children, Western Medicine
\end{abstract}

\begin{abstract}
Objective: Analysis xiaoqinglong decoction with gypsum decoction clinical efficacy of treatment of children with pneumonia. Method; pneumonia in this study between May 2014 May 2015 in our hospital treated 100 cases, according to the different treatment methods will be divided into a control group ( $\mathrm{n}=50$ patients) and observation group ( $=50$ cases) and control group were treated with western medicine, that give children with anti-infection and anti-inflammatory treatment, to give children the treatment group were observed in the combination therapy methods of treatment of children with pneumonia were compared. Results; children observation group total effective rate after combination therapy was significantly higher than $96 \%$ using the total efficiency of $88 \%$ after only western medicine, significant difference was statistically significant $(\mathrm{p}<0.05)$.. After the observation group were treated with combination therapy of cough, rales, sputum, shortness of breath, shortness of breath improved significantly Friendship control group, the difference was significant, statistically significant $(\mathrm{p}<0.05)$. Conclusion; children with pneumonia in children with the use of Western medicine xiaoqinglong decoction with gypsum decoction treatment, the effect is significant.
\end{abstract}

\section{Introduction}

Pneumonia in children under 5 years old infants a common multiple inflammatory lung disease, the main cause is that a variety of pathogens, or a variety of factors caused infant lung inflammation [1]. Related survey, the disease has a high mortality rate, which is the main cause of pneumonia deaths. So effectively strengthen the treatment of pneumonia in children is particularly important. The study used in combination therapy method of treating pneumonia, and achieved satisfactory results, are summarized below.

\section{Subjects and Methods}

The Study. The study between May 2014 May 2015 in our hospital treated 100 cases of pneumonia in children, all patients in this study are in line with "Zhu Fu Tang Practical Pediatrics," the diagnostic criteria for this study 100 patients showed varying degrees of cough, sputum expectoration, fine wet rales, wheezing, fever. According to the different treatment methods will be divided into a control group ( $\mathrm{n}=50$ patients) and observation group ( $\mathrm{n}=50$ patients), patients in the observation group and 27 females, 23 males; pneumonia youngest of five months, maximum age 7 years old, with a median age (4.2 \pm 1.3$)$ years; where children eight cases of pneumonia, 14 cases of bronchiolitis, pneumonia and high fever in children with 28 cases; the control group of patients, 26 were women, 24 males; advanced colon cancer patients aged 6 months minimum, maximum 7 years old, with a median age $(4.6 \pm 1.1)$ years; where the children seven cases of pneumonia, 16 cases of bronchiolitis, 27 cases of children with high fever pneumonia. All types of diseases object of study, age, sex and other baseline data, through statistical analysis, not statistically significant. Comparable is $\mathrm{p}>0.05$.

Treatment. The control group were treated with western medicine, that give children with 
anti-infection and anti-inflammatory treatment, according to the condition of the infants received sodium in the treatment at a dose of $50 \mathrm{mg} / \mathrm{kg}$ per day, divided into 3 doses; if the children had a viral infection, then give children with ribavirin at a dose of 10mg / $\mathrm{kg}$ per day, 1 doses, and can add or subtract the dose according to the actual conditions of children, the maximum dose of $15 \mathrm{mg} / \mathrm{kg}$ per day, children pay close attention to quality water and electricity balance during treatment according to the actual condition of children with symptomatic and supportive treatment for 7 days of treatment.

Asarum 2g, 6g ginger, 8g plaster, 6g Gardenia: administering the treatment of children with the observation group, the treatment of children in the control group based on the use of xiaoqinglong decoction with gypsum decoction treatment, prescription use by the method of Integrative Medicine , 3g ephedra, 3g berberine, 6g Schisandra, 10g skullcap, 3g licorice, 5g Cork, 6g France Breit, 6g fragrant soy, 6g Guizhi, 8g peony composition, based on age, weight and condition of the children was the right amount of state adjust the dose; simultaneously flavored drugs symptomatic children, and children with thick yellow phlegm symptoms, prescription Canada Fritillaria, melon baskets, while France Breit's prescription dose to 10g; children the presence of symptoms of shortness of breath, prescription Tinglizi added $6 \mathrm{~g}$ and $8 \mathrm{~g}$ loquat leaf; children with strong heat, thirst or sweating symptoms, prescriptions plus $6 \mathrm{~g}$ altogether, while the plaster prescription dose to $15 \mathrm{~g}$; the presence of children with constipation symptoms, prescriptions plus $3 \mathrm{~g}$ rhubarb. Said prescription one day, with clear water simmer in water, 7 days continuous treatment effects were observed in the two groups of children with pneumonia.

Outcome Measures. Observed and compared the two groups of children with pneumonia sputum, coughing, wheezing and other symptoms politics, and wet and dry rales time the temperature drops.

Determine the Efficacy. Cure; after treatment, body temperature returned to normal in children with pneumonia, pulmonary disappeared, symptoms, radiographic X-ray examination showed pulmonary lesions completely absorbed blood as normal. Better; after treatment, the body temperature of children with pneumonia before treatment significantly improved pulmonary rales decreased symptoms, radiographic X-ray examination showed lung lesions are not completely absorbed or partially absorbed. Invalid no significant changes in symptoms and signs, or signs of increase. The total effective rate $=($ cure + improvement $) /$ total cases $\times 100 \%$.

Statistical Methods. The collected set of experimental data, statistical software for data processing in data package SPSS20.0, count data and measurement data were applied (n\%) and ( $\square \mathrm{x} \pm \mathrm{s}$ ) that the differences between the groups by $\mathrm{x}^{2}$ and t-test, when $\mathrm{P}<0.05$, the difference was statistically significant.

\section{Results Study}

Comparison of two Groups of Children with Clinical Efficacy. The total group of children observed after using the combination therapy was significantly higher efficiency of $96 \%$ using the total efficiency of $88 \%$ after western medicine alone., A significant difference was statistically significant $(\mathrm{p}<0.05)$. Table 1.

Table 1 Comparison of two groups of children with clinical efficacy [n (\%)]

\begin{tabular}{llllll}
\hline Group & Case & OK $(\mathrm{n})$ & Improve $(\mathrm{n})$ & NG $(\mathrm{n})$ & Ration (\%) \\
\hline Observer Gr. & 50 & $39(78.00)$ & $9(18.00)$ & $2(4.00)$ & 96.00 \\
Comparative Gr. & 50 & $31(62.00)$ & $13(26.00)$ & $6(12.00)$ & 88.00 \\
X2 & & 6.0952 & 1.8648 & 4.3478 & 4.3478 \\
p & & 0.0135 & 0.1721 & 0.0000 & 0.0000
\end{tabular}

The Two Groups Of Children With Clinical Symptoms And Signs Of Recovery Time Time Comparison. After the observation group were treated with combination therapy of cough, rales, 
sputum, shortness of breath, shortness of breath improved significantly Friendship control group, the difference was significant, statistically significant ( $<<0.05$ ). Table 2.

Table 2 groups of children with clinical symptoms and signs of recovery time comparison ( $\square \mathrm{x} \pm$

s)

\begin{tabular}{lcccccc}
\hline Group & Case & cough (d) & $\begin{array}{l}\text { Expectoratio } \\
\mathrm{n}(\mathrm{d})\end{array}$ & $\begin{array}{l}\text { Shortness of } \\
\text { breath }(\mathrm{d})\end{array}$ & $\begin{array}{l}\text { Shortness of } \\
\text { breath }(\mathrm{d})\end{array}$ & Rales $(\mathrm{d})$ \\
\hline Observer Gr. & 50 & $7.3 \pm 1.4$ & $6.0 \pm 2.2$ & $3.2 \pm 2.6$ & $3.1 \pm 2.4$ & $4.6 \pm 1.3$ \\
Comparative & 50 & $9.5 \pm 1.9$ & $7.3 \pm 2.6$ & $4.3 \pm 2.1$ & $4.7 \pm 2.9$ & $6.5 \pm 1.2$ \\
Gr. & & 5.8956 & 2.4140 & 2.0816 & 2.6882 & 6.7922 \\
X2 & 0.0000 & 0.0181 & 0.0407 & 0.0088 & 0.0000 \\
p & & & & & &
\end{tabular}

\section{Discussion}

Children Pneumonia is a common clinical multiple a lung inflammation disease, the main cause of infant lung inflammation that various pathogens or a variety of factors caused. If not timely and effective treatment, it will seriously affect the normal development of infants and young children, and even lead to death in children [3]. In the clinical treatment of Western medicine mainly for anti-inflammatory, anti-infective and anti-viral treatment, the initial phase of the treatment effect is very good, but the overall treatment effect is unsatisfactory. Chinese medicine believes that children with pneumonia are "febrile disease" and "cough" category. The main causes that children with exogenous cold caused by wind-heat evil, since evil and into the body through the nose and mouth of children, and then the invasion of the lung, eventually leading to the occurrence of cough [4]. And because infants belong to Zhi Yin, Zhi Yang body, the infant population more susceptible to heat cold wind evil invasion, but also distinguish the cold and wind heat Chinese medicine clinical main reason [5].

The main role of efficacy xiaoqinglong decoction is relieving cold, warm the lung casual drink, to drink Chinese medicine treatment commonly prescribed evil and pathogenic cold outside; prescription and ginger Asarum Chinese medicine prescriptions used to treat the drink temperature the main drugs lungs, in addition to good effect Guizhi solution table; and Guizhi ephedra and has a relatively good asthma relieving cold effect; prescriptions can effectively help children with Pinellia and stomach expectorant Sanjie; Guizhi peony and having to reconcile business health effect; Schisandra convergence lung and effective cough efficacy me, gypsum Big Chill is the drugs get into the lung and stomach by having a good clear lung heat effect; and berberine, skullcap , Cork, gardenia together, having a good effect purging fire detoxification. The study showed that the observation group were treated with total efficiency for the combination therapy was significantly higher than $96 \%$ after using the total efficiency of $88 \%$ western medicine alone., Described Western Medicine xiaoqinglong decoction with gypsum decoction treatment of pneumonia in children with a significant effect [6].

Overall, children with pneumonia in children using western xiaoqinglong decoction with gypsum decoction treatment, the effect is significant.

\section{References}

[1] Zhen Renshen. Xiaoqinglong decoction addition and subtraction of clinical studies in children with pneumonia [J]. Henan TCM treatment, 2016, 36 (2): 204-206.

[2] Gao Huiping .Xiaoqinglong decoction with gypsum decoction clinical efficacy of treatment of children with pneumonia [J]. Chemists (Academic Edition), 2012, 10 (11): 842 
[3] Li Boning. Clinical efficacy of Xiaoqinglong decoction with gypsum decoction treatment of children with pneumonia was observed [J]. TCM Clinical Research, 2016, (3): 69-70.

[4] Wu Yulin. The clinical efficacy of xiaoqinglong decoction with gypsum decoction treatment of children with pneumonia was observed [J]. Inner Mongolia Medicine, 2015, 34 (12): 6-7.

[5] Tang Huhai. Fu Yucheng. Integrative Medicine Pneumonia in Children observation [J]. Practical Traditional Chinese Medicine, 2016, 32 (7): 685-685.

[6] Tian Li, Gao Ya, Zheng Chunyan, etc. Zhou Lin spectrum analyzer with internal medicine treatment of children recovering from pneumonia 30 cases [J]. Traditional Chinese Medicine Research, 2012, 25 (1): 37-38. 